\title{
SOME RESULTS ON THE JOINT HIGHER NUMERICAL RANGES AND RADII OF MATRICES
}

\author{
GHOLAMREZA AGHAMOLLAEI AND MOHAMMAD ALI NOUROLLAHI
}

Received 18 September, 2015

\begin{abstract}
In this paper, some algebraic properties of the joint $k$-numerical radius, joint $k-$ radius and the joint $k$-norm of matrices are investigated. Moreover, using the joint higher numerical ranges of diagonal matrices which are convex polyhedrons, a description for the shape of the higher numerical ranges of matrix polynomials is given.
\end{abstract}

2010 Mathematics Subject Classification: 15A60; 15A18; 47A56

Keywords: joint $k$-numerical range, joint $k$-numerical radius, joint $k$-norm, matrix polynomial, norms

\section{INTRODUCTION AND PRELIMINARIES}

Let $\mathrm{M}_{n \times m}$ be the vector space of all $n \times m$ complex matrices. For the case $n=$ $m, \mathrm{M}_{n \times n}$ is denoted by $\mathrm{M}_{n}$; namely, the algebra of all $n \times n$ complex matrices. Throughout the paper, $k, m$ and $n$ are considered as positive integers, and $k \leq n$. Moreover, $I_{k}$ denotes the $k \times k$ identity matrix. The set of all $n \times k$ isometry matrices is denoted by $X_{n \times k}$; i.e., $X_{n \times k}=\left\{X \in \mathbb{M}_{n \times k}: X^{*} X=I_{k}\right\}$. Also, the group of $n \times n$ unitary matrices is denoted by $U_{n}$; namely,

$$
U_{n}=\left\{U \in \mathbb{M}_{n}: U^{*} U=I_{n}\right\}=X_{n \times n} .
$$

The notion of $k$-numerical range of $A \in \mathrm{M}_{n}$, which was first introduced by P. R. Halmos in [8], is defined and denoted by

$$
W_{k}(A)=\left\{\frac{1}{k} \operatorname{tr}\left(X^{*} A X\right): X \in X_{n \times k}\right\},
$$

where $\operatorname{tr}($.$) denotes the trace. The sets W_{k}(A)$, where $k \in\{1,2, \ldots, n\}$, are generally called the higher numerical ranges of $A$. When $k=1$, we have the classical numerical range $W_{1}(A)=W(A)=\left\{x^{*} A x: x \in \mathbb{C}^{n}, x^{*} x=1\right\}$, which has been studied extensively; see for example [7] and [9, Chapter 1]. Motivation of our study comes from finite-dimensional quantum systems. In quantum physics, e.g., see [6], the quantum states are represented by density matrices, i.e., positive semidefinite matrices with trace one. If a quantum state $D \in \mathbb{M}_{n}$ has rank one, i.e., $D=x x^{*}$ 
for some $x \in \mathbb{C}^{n}$ with $x^{*} x=1$, then $D$ is called a pure quantum state; otherwise, $D$ is said to be a mixed quantum state which can be written as a convex combination of pure quantum states. So, for $A \in \mathbb{M}_{n}$, we have $W(A)=\{\operatorname{tr}(A D): D \in$ $\mathrm{M}_{n}$ is a pure quantum state\}. It is known that $A$ is Hermitian if and only if $W(A) \subseteq \mathbb{R}$, and also $A$ is a positive semidefinite matrix if and only if $W(A) \subseteq[0, \infty]$. Moreover, by the fact that the convex hull of the set

$$
\left\{\frac{1}{k} P: P \in \mathbb{M}_{n} \text { is Hermitian, } P^{2}=P \text {, and } \operatorname{tr}(P)=k\right\}
$$

equals to the set $\delta_{k}$ of density matrices $D \in \mathrm{M}_{n}$ satisfying $\frac{1}{k} I_{n}-D$ is positive semidefinite, we have

$$
\begin{aligned}
W_{k}(A) & =\left\{\frac{1}{k} \operatorname{tr}(A P): P \in \mathbb{M}_{n}, P^{2}=P=P^{*}, \operatorname{tr}(P)=k\right\} \\
& =\left\{\operatorname{tr}(A D): D \in 8_{k}\right\} .
\end{aligned}
$$

Let $A \in \mathbb{M}_{n}$ have eigenvalues $\lambda_{1}, \lambda_{2}, \ldots, \lambda_{n}$, counting multiplicities. The set of all $k$-averages of eigenvalues of $A$ is denoted by $\sigma^{(k)}(A)$; namely,

$$
\sigma^{(k)}(A)=\left\{\frac{1}{k}\left(\lambda_{i_{1}}+\lambda_{i_{2}}+\cdots+\lambda_{i_{k}}\right): 1 \leq i_{1}<i_{2}<\cdots<i_{k} \leq n\right\} .
$$

Notice that if $k=1$, then $\sigma^{(1)}(A)=\sigma(A)$, i.e., the spectrum of $A$. Next, we list some properties of the $k$-numerical range of matrices which will be useful in our discussion. For more details, see $[8,13]$ and their references.

Proposition 1. Let $A \in \mathrm{M}_{n}$. Then the following assertions are true:

(i) $W_{k}(A)$ is a compact and convex set in $\mathbb{C}$;

(ii) $\operatorname{conv}\left(\sigma^{(k)}(A)\right) \subseteq W_{k}(A)$, where conv( $\left.S\right)$ denotes the convex hull of a set $S \subseteq \mathbb{C}$. The equality holds if $A$ is normal;

(iii) $\left\{\frac{1}{n} \operatorname{tr}(A)\right\}=W_{n}(A) \subseteq W_{n-1}(A) \subseteq \cdots \subseteq W_{2}(A) \subseteq W_{1}(A)=W(A)$;

(iv) If $V \in \mathcal{X}_{n \times s}$, where $k \leq s \leq n$, then $W_{k}\left(V^{*} A V\right) \subseteq W_{k}(A)$. The equality holds if $s=n$, i.e., $W_{k}\left(U^{*} A U\right)=W_{k}(A)$, where $U \in U_{n}$;

(v) For any $\alpha, \beta \in \mathbb{C}, W_{k}\left(\alpha A+\beta I_{n}\right)=\alpha W_{k}(A)+\beta$, and for the case $k<n$, $W_{k}(A)=\{\alpha\}$ if and only if $A=\alpha I_{n}$.

Let $A \in \mathrm{M}_{n}$. The $k$-numerical radius, $k$-spectral radius and the $k$-spectral norm of $A$ are defined and denoted, respectively, by

and

$$
\begin{aligned}
r_{k}(A) & :=\max \left\{|z|: z \in W_{k}(A)\right\}, \\
\rho_{k}(A) & :=\max \left\{|z|: z \in \sigma^{(k)}(A)\right\},
\end{aligned}
$$

$$
\|A\|_{(k)}:=\max \left\{\left|\frac{1}{k} \operatorname{tr}\left(X^{*} A Y\right)\right|: X, Y \in X_{n \times k}\right\} .
$$

It is clear that $r_{1}(A)=r(A), \rho_{1}(A)=\rho(A)$, and $\|A\|_{(1)}=\|A\|$, which are the numerical radius, the spectral radius, and the spectral matrix norm (i.e., the matrix norm 
subordinate to the Euclidean vector norm) of $A$, respectively. Also, $r_{k}($.$) for the case$ $k<n$, and $\|.\|_{(k)}$ are vector norms on $\mathbf{M}_{n}$. Now, in the following proposition, we state some other properties of $\rho_{k}(),. r_{k}($.$) and \|.\|_{(k)}$, and their relations. For more details, see $[10,12]$.

Proposition 2. Let $A \in \mathrm{M}_{n}$. Then the following assertions are true:

(i) $r_{k}\left(V^{*} A V\right) \leq r_{k}(A)$ and $\left\|V^{*} A V\right\|_{(k)} \leq\|A\|_{(k)}$ for all $V \in \mathcal{X}_{n \times s}$, where $k \leq s \leq n$. The equality holds if $s=n$;

(ii) $\|A\|_{(k)}=\frac{1}{k} \sum_{i=1}^{k} s_{i}(A)$, where $s_{1}(A) \geq s_{2}(A) \geq \cdots \geq s_{n}(A)$ are the singular values of $A$;

(iii) $\rho_{k}(A) \leq r_{k}(A) \leq\|A\|_{(k)}$;

(iv) $r_{k}(A)=\|A\|_{(k)}$ if and only if there exists a number $\theta \in \mathbb{R}$ such that $\alpha_{j}=$ $a_{j} e^{i \theta}$ for all $j=1,2, \ldots, k$, where $a_{1} \geq a_{2} \geq \cdots \geq a_{n}$ are the singular values and $\alpha_{1}, \alpha_{2}, \ldots, \alpha_{n}$, where $\left|\alpha_{1}\right| \geq\left|\alpha_{2}\right| \geq \cdots \geq\left|\alpha_{n}\right|$, are the eigenvalues of $A$;

(v) If $k<n$, then $\frac{1}{2(2 k-1)}\|A\| \leq r_{k}(A) \leq\|A\|$;

(vi) $\|A\|_{(n)} \leq\|A\|_{(n-1)} \leq \cdots \leq\|A\|_{(1)}=\|A\|$.

In Section 2, we study some algebraic properties of the joint $k$-numerical radius, joint $k$-spectral radius and the joint $k$-norm of matrices. In Section 3 , using the joint higher numerical ranges of diagonal matrices which are convex polyhedrons, we give a description for the shape of the higher numerical ranges of matrix polynomials.

\section{MAIN RESULTS}

We begin this section by introducing the notion of the joint $k$-numerical radius of matrices.

Definition 1. Let $\left(A_{1}, A_{2}, \ldots, A_{m}\right) \in \mathbb{M}_{n}^{m}$. The joint $k$-numerical radius of $\left(A_{1}, A_{2}, \ldots, A_{m}\right)$ is defined and denoted by

$$
\begin{aligned}
r_{k}\left(A_{1}, A_{2}, \ldots, A_{m}\right):=\sup \{ & l_{2}\left(a_{1}, a_{2}, \ldots, a_{m}\right) \\
& \left.:\left(a_{1}, a_{2}, \ldots, a_{m}\right) \in W_{k}\left(A_{1}, A_{2}, \ldots, A_{m}\right)\right\},
\end{aligned}
$$

where

$$
\begin{aligned}
l_{2}\left(a_{1}, a_{2}, \ldots, a_{m}\right) & =\left(\sum_{i=1}^{m}\left|a_{i}\right|^{2}\right)^{\frac{1}{2}}, \text { and } \\
W_{k}\left(A_{1}, A_{2}, \ldots, A_{m}\right) & =\left\{\left(\frac{1}{k} \operatorname{tr}\left(X^{*} A_{1} X\right), \ldots, \frac{1}{k} \operatorname{tr}\left(X^{*} A_{m} X\right)\right): X \in \mathcal{X}_{n \times k}\right\}
\end{aligned}
$$

is the joint $k$-numerical range of $\left(A_{1}, A_{2}, \ldots, A_{m}\right)$.

Next, we are going to state a new description of $r_{k}\left(A_{1}, A_{2}, \ldots, A_{m}\right)$ which is one of the our main results in this section. For this, we need the following lemma. 
Lemma 1. Let $\left(a_{1}, a_{2}, \ldots, a_{m}\right) \in \mathbb{C}^{m}$. Then

$$
l_{2}\left(a_{1}, a_{2}, \ldots, a_{m}\right)=\sup _{\left(\lambda_{1}, \lambda_{2}, \ldots, \lambda_{m}\right) \in S^{1}}\left|\sum_{j=1}^{m} \lambda_{j} a_{j}\right|,
$$

where $S^{1}=\left\{\left(z_{1}, z_{2}, \ldots, z_{m}\right) \in \mathbb{C}^{m}: \sum_{i=1}^{m}\left|z_{i}\right|^{2}=1\right\}$.

Proof. To avoid of trivial case, we assume that $\left(a_{1}, a_{2}, \ldots, a_{m}\right) \neq(0,0, \ldots, 0)$. Let $\left(\lambda_{1}, \lambda_{2}, \ldots, \lambda_{m}\right) \in S^{1}$ be arbitrary. By the Cauchy-Schwartz inequality, we have

$$
\left|\sum_{i=1}^{m} \lambda_{i} a_{i}\right| \leq\left(\sum_{i=1}^{m}\left|\lambda_{i}\right|^{2}\right)^{\frac{1}{2}}\left(\sum_{i=1}^{m}\left|a_{i}\right|^{2}\right)^{\frac{1}{2}}=l_{2}\left(a_{1}, a_{2}, \ldots, a_{m}\right) .
$$

So,

$$
\sup _{\left(\lambda_{1}, \lambda_{2}, \ldots, \lambda_{m}\right) \in S^{1}}\left|\sum_{j=1}^{m} \lambda_{j} a_{j}\right| \leq l_{2}\left(a_{1}, a_{2}, \ldots, a_{m}\right) .
$$

By setting $\lambda_{i}=\frac{\overline{a_{i}}}{l_{2}\left(a_{1}, \ldots, a_{m}\right)}$, where $i=1,2, \ldots, m$, we have $\left(\lambda_{1}, \lambda_{2}, \ldots, \lambda_{m}\right) \in S^{1}$. Moreover,

$$
\left|\sum_{j=1}^{m} \lambda_{j} a_{j}\right|=\frac{\sum_{j=1}^{m}\left|a_{j}\right|^{2}}{l_{2}\left(a_{1}, a_{2}, \ldots, a_{m}\right)}=l_{2}\left(a_{1}, a_{2}, \ldots, a_{m}\right) .
$$

Therefore, the result holds.

Theorem 1. If $\left(A_{1}, A_{2}, \ldots, A_{m}\right) \in \mathbb{M}_{n}^{m}$, then

$$
r_{k}\left(A_{1}, A_{2}, \ldots, A_{m}\right)=\sup _{\left(\lambda_{1}, \lambda_{2}, \ldots, \lambda_{m}\right) \in S^{1}} r_{k}\left(\lambda_{1} A_{1}+\lambda_{2} A_{2}+\cdots+\lambda_{m} A_{m}\right) .
$$

Proof. For every $\left(\lambda_{1}, \lambda_{2}, \ldots, \lambda_{m}\right) \in S^{1}$, we have

$$
\begin{aligned}
& W_{k}\left(\lambda_{1} A_{1}+\lambda_{2} A_{2}+\cdots+\lambda_{m} A_{m}\right)=\left\{\frac{1}{k} \operatorname{tr}\left(X^{*}\left(\sum_{j=1}^{m} \lambda_{j} A_{j}\right) X\right): X \in X_{n \times k}\right\} \\
& =\left\{\sum_{j=1}^{m} \lambda_{j} a_{j}:\left(a_{1}, a_{2}, \ldots, a_{m}\right) \in W_{k}\left(A_{1}, A_{2}, \ldots, A_{m}\right)\right\} .
\end{aligned}
$$

Thus,

$$
\begin{aligned}
r_{k}\left(\lambda_{1} A_{1}+\lambda_{2} A_{2}\right. & \left.+\cdots+\lambda_{m} A_{m}\right) \\
& =\sup \left\{\left|\sum_{j=1}^{m} \lambda_{j} a_{j}\right|:\left(a_{1}, a_{2}, \ldots, a_{m}\right) i n W_{k}\left(A_{1}, A_{2}, \ldots, A_{m}\right)\right\} .
\end{aligned}
$$


Now, using Definition 1 and Lemma 1, we have:

$$
\begin{aligned}
r_{k}\left(A_{1}, A_{2}, \ldots, A_{m}\right) & =\sup _{\left(a_{1}, a_{2}, \ldots, a_{m}\right) \in W_{k}\left(A_{1}, A_{2}, \ldots, A_{m}\right)} l_{2}\left(a_{1}, a_{2}, \ldots, a_{m}\right) \\
& =\sup _{\left(a_{1}, a_{2}, \ldots, a_{m}\right) \in W_{k}\left(A_{1}, A_{2}, \ldots, A_{m}\right)} \sup _{\left(\lambda_{1}, \lambda_{2}, \ldots, \lambda_{m}\right) \in S^{1}}\left|\sum_{j=1}^{m} \lambda_{j} a_{j}\right| \\
& =\sup _{\left(\lambda_{1}, \lambda_{2}, \ldots, \lambda_{m}\right) \in S^{1}} \sup _{\left(a_{1}, a_{2}, \ldots, a_{m}\right) \in W_{k}\left(A_{1}, A_{2}, \ldots, A_{m}\right)}\left|\sum_{j=1}^{m} \lambda_{j} a_{j}\right| \\
& =\sup _{\left(\lambda_{1}, \lambda_{2}, \ldots, \lambda_{m}\right) \in S^{1}} r_{k}\left(\lambda_{1} A_{1}+\lambda_{2} A_{2}+\ldots+\lambda_{m} A_{m}\right) .
\end{aligned}
$$

So, the proof is complete.

In the following proposition which follows from Theorem 1 and Propositions 1 and 2, we state some basic properties of the joint $k$-numerical radius of matrices.

Proposition 3. Let $\left(A_{1}, A_{2}, \ldots, A_{m}\right) \in \mathbb{M}_{n}^{m}$. Then the following assertions are true:

(i) If $k<n$, then $r_{k}\left(A_{1}, A_{2}, \ldots, A_{m}\right)=0$ if and only if $A_{1}=A_{2}=\cdots$ $=A_{m}=0$;

(ii) $r_{k}\left(\lambda A_{1}, \lambda A_{2}, \ldots, \lambda A_{m}\right)=|\lambda| r_{k}\left(A_{1}, A_{2}, \ldots, A_{m}\right)$ for all $\lambda \in \mathbb{C}$;

(iii) $r_{k}\left(A_{1}+A_{1}^{\prime}, \ldots, A_{m}+A_{m}^{\prime}\right) \leq r_{k}\left(A_{1}, \ldots, A_{m}\right)+r_{k}\left(A_{1}^{\prime}, \ldots, A_{m}^{\prime}\right)$, where $A_{1}^{\prime}, A_{2}^{\prime}, \ldots, A_{m}^{\prime} \in \mathrm{M}_{n}$

(iv) $r_{k}\left(S^{*} A_{1} S, S^{*} A_{2} S, \ldots, S^{*} A_{m} S\right) \leq r_{k}\left(A_{1}, A_{2}, \ldots, A_{m}\right)$ for all $S \in \mathcal{X}_{n \times t}$, where $k \leq t \leq n$. The equality holds if $t=n$. Consequently, for every $U \in U_{n}$

$$
r_{k}\left(U^{*} A_{1} U, U^{*} A_{2} U, \ldots, U^{*} A_{m} U\right)=r_{k}\left(A_{1}, A_{2}, \ldots, A_{m}\right) .
$$

In view of Proposition $3, r_{k}(., \ldots,$.$) , where k<n$, is a vector norm on $\mathbf{M}_{n}^{m}$. Moreover, Theorem 1 leads us to introduce the notion of the joint $k$-norm of matrices.

Definition 2. Let $\left(A_{1}, \ldots, A_{m}\right) \in \mathbb{M}_{n}^{m}$. The joint $k$-norm of $\left(A_{1}, \ldots, A_{m}\right)$ is defined and denoted by

$$
\left\|\left(A_{1}, A_{2}, \ldots, A_{m}\right)\right\|_{(k)}:=\sup _{\left(\lambda_{1}, \lambda_{2}, \ldots, \lambda_{m}\right) \in S^{1}}\left\|\lambda_{1} A_{1}+\lambda_{2} A_{2}+\cdots+\lambda_{m} A_{m}\right\|_{(k)} .
$$

Using Definition 2 and Proposition 2, we can show that the joint $k$-norm of matrices satisfies in the following basic properties.

Proposition 4. Let $\left(A_{1}, A_{2}, \ldots, A_{m}\right) \in \mathbb{M}_{n}^{m}$. Then the following assertions are true:

(i) If $k<n$, then $\left\|\left(A_{1}, A_{2}, \ldots, A_{m}\right)\right\|_{(k)}=0$ if and only if $A_{1}=A_{2}=\cdots$ $=A_{m}=0$; 
(ii) $\left\|\left(\lambda A_{1}, \lambda A_{2}, \ldots, \lambda A_{m}\right)\right\|_{(k)}=|\lambda|\left\|\left(A_{1}, A_{2}, \ldots, A_{m}\right)\right\|_{(k)}$ for all $\lambda \in \mathbb{C}$;

(iii) $\left\|\left(A_{1}+A_{1}^{\prime}, \ldots, A_{m}+A_{m}^{\prime}\right)\right\|_{(k)} \leq\left\|\left(A_{1}, \ldots, A_{m}\right)\right\|_{(k)}+\left\|\left(A_{1}^{\prime}, \ldots, A_{m}^{\prime}\right)\right\|_{(k)}$, where $\left(A_{1}^{\prime}, A_{2}^{\prime}, \ldots, A_{m}^{\prime}\right) \in \mathbb{M}_{n}^{m}$;

(iv) $\left\|\left(S^{*} A_{1} S, S^{*} A_{2} S, \ldots, S^{*} A_{m} S\right)\right\|_{(k)} \leq\left\|\left(A_{1}, A_{2}, \ldots, A_{m}\right)\right\|_{(k)}$ for all $S \in \mathcal{X}_{n \times t}$, where $k \leq t \leq n$. The equality is holds if $t=n$. Consequently, for every $U \in \mathcal{U}_{n}$,

$$
\left\|\left(U^{*} A_{1} U, U^{*} A_{2} U, \ldots, U^{*} A_{m} U\right)\right\|_{(k)}=\left\|\left(A_{1}, A_{2}, \ldots, A_{m}\right)\right\|_{(k)} .
$$

In view of Proposition 4, for the case $k<n$, the joint $k$-norm is a vector norm on $\mathrm{M}_{n}^{m}$. In the final result of this section, we are going to study the relations between the joint $k$-numerical radius and the joint $k$-norm of matrices. For this, we need the following lemma.

Lemma 2. Let $A \in \mathbb{M}_{n}$. Then

$$
\frac{1}{2(2 k-1)}\|A\|_{(k)} \leq r_{k}(A) \leq\|A\|_{(k)} .
$$

Proof. The first inequality follows from Proposition $2((v)$ and $(v i))$. Also, by (1.1) and (1.3), it is clear that $r_{k}(A) \leq\|A\|_{(k)}$, and so, the proof is complete.

Theorem 2. Let $\left(A_{1}, A_{2}, \ldots, A_{m}\right) \in \mathbb{M}_{n}^{m}$. Then

$$
\begin{aligned}
\frac{1}{2(2 k-1)}\left\|\left(A_{1}, \ldots, A_{m}\right)\right\|_{(k)} & \leq r_{k}\left(A_{1}, \ldots, A_{m}\right) \\
& \leq \min \left\{\left\|\left(A_{1}, \ldots, A_{m}\right)\right\|_{(k)}, l_{2}\left(r_{k}\left(A_{1}\right), \ldots, r_{k}\left(A_{m}\right)\right)\right\} .
\end{aligned}
$$

Moreover, the right inequality is sharp.

Proof. Applying Lemma 2 to the matrix $A:=\lambda_{1} A_{1}+\lambda_{2} A_{2}+\cdots+\lambda_{m} A_{m}$, where $\left(\lambda_{1}, \lambda_{2}, \ldots, \lambda_{m}\right) \in S^{1}$, and using Definition 2 and Theorem 1, we have

$$
\frac{1}{2(2 k-1)}\left\|\left(A_{1}, \ldots, A_{m}\right)\right\|_{(k)} \leq r_{k}\left(A_{1}, \ldots, A_{m}\right) \leq\left\|\left(A_{1}, \ldots, A_{m}\right)\right\|_{(k)} .
$$

Moreover, it is clear that $W_{k}\left(A_{1}, A_{2}, \ldots, A_{m}\right) \subseteq W_{k}\left(A_{1}\right) \times W_{k}\left(A_{2}\right) \times \cdots \times W_{k}\left(A_{m}\right)$. Then,

$$
\begin{aligned}
r_{k}\left(A_{1}, \ldots, A_{m}\right) & =\sup \left\{l_{2}\left(a_{1}, \ldots, a_{m}\right):\left(a_{1}, \ldots, a_{m}\right) \in W_{k}\left(A_{1}, \ldots, A_{m}\right)\right\} \\
& \leq l_{2}\left(r_{k}\left(A_{1}\right), \ldots, r_{k}\left(A_{m}\right)\right) .
\end{aligned}
$$

Therefore, the right inequality also holds.

To prove that the right inequality is sharp, we consider the matrix $A \in \mathbb{M}_{n}$ with singular values $a_{1} \geq a_{2} \geq \cdots \geq a_{n}$ and eigenvalues $\alpha_{1}, \alpha_{2}, \ldots, \alpha_{n}$, where $\left|\alpha_{1}\right| \geq\left|\alpha_{2}\right| \geq$ $\cdots \geq\left|\alpha_{n}\right|$. Moreover, we assume that there exist a $\theta \in \mathbb{R}$ such that $\alpha_{j}=a_{j} e^{i \theta}$ for all $j=1,2, \ldots, n$. Then by Proposition 2(v), we have $r_{k}(A)=\|A\|_{(k)}$. Now, suppose 
that $A_{j}=t_{j} A$, where $t_{j} \in \mathbb{C}$ for all $j=1,2, \ldots, m$. Then by Theorem 1, Proposition 4 and Definition 2, we have:

$$
\begin{aligned}
r_{k}\left(A_{1}, A_{2}, \ldots, A_{m}\right) & =\sup _{\left(\lambda_{1}, \lambda_{2}, \ldots, \lambda_{m}\right) \in S^{1}} r_{k}\left(\lambda_{1} A_{1}+\lambda_{2} A_{2}+\cdots+\lambda_{m} A_{m}\right) \\
& =\sup _{\left(\lambda_{1}, \lambda_{2}, \ldots, \lambda_{m}\right) \in S^{1}}\left|\sum_{j=1}^{m} t_{j} \lambda_{j}\right| r_{k}(A) \\
& =\sup _{\left(\lambda_{1}, \lambda_{2}, \ldots, \lambda_{m}\right) \in S^{1}}\left|\sum_{j=1}^{m} t_{j} \lambda_{j}\right|\|A\|_{(k)} \\
& =\sup _{\left(\lambda_{1}, \lambda_{2}, \ldots, \lambda_{m}\right) \in S^{1}}\left\|\lambda_{1} A_{1}+\lambda_{2} A_{2}+\cdots+\lambda_{m} A_{m}\right\|_{(k)} \\
& =\left\|\left(A_{1}, A_{2}, \ldots, A_{m}\right)\right\|_{(k)} .
\end{aligned}
$$

Now, if we choose $t_{j} \geq 0$, then by Lemma 1 and the above inequalities, we have:

$$
\left\|\left(A_{1}, A_{1}, \ldots, A_{m}\right)\right\|_{(k)}=l_{2}\left(r_{k}\left(A_{1}\right), r_{k}\left(A_{2}\right), \ldots, r_{k}\left(A_{m}\right)\right) .
$$

Hence, the right inequality changes to equality. This completes the proof.

\section{AdDitional RESUlts}

In this section, by using the joint higher numerical ranges of diagonal matrices, we find an approximation for the higher numerical ranges of matrix polynomials. For this, suppose that

$$
P(\lambda)=A_{m} \lambda^{m}+A_{m-1} \lambda^{m-1}+\cdots+A_{1} \lambda+A_{0}
$$

is a matrix polynomial, where $A_{i} \in \mathbb{M}_{n}(i=0,1, \ldots, m), A_{m} \neq 0$ and $\lambda$ is a complex variable. The numbers $m$ and $n$ are referred as the degree and the order of $P(\lambda)$, respectively. The matrix polynomial $P(\lambda)$ is said to be a diagonal matrix polynomial if all the coefficients $A_{i}$ are diagonal matrices. A scalar $\lambda_{0} \in \mathbb{C}$ is called an eigenvalue of $P(\lambda)$ if the system $P\left(\lambda_{0}\right) x=0$ has a nonzero solution $x_{0} \in \mathbb{C}^{n}$. This solution $x_{0}$ is known as an eigenvector of $P(\lambda)$ corresponding to $\lambda_{0}$, and the set of all eigenvalues of $P(\lambda)$ is said to be the spectrum of $P(\lambda)$; namely,

$$
\sigma[P(\lambda)]=\{\mu \in \mathbb{C}: \operatorname{det}(P(\mu))=0\} .
$$

The (classical) numerical range of $P(\lambda)$ is defined and denoted by

$$
W[P(\lambda)]:=\left\{\mu \in \mathbb{C}: x^{*} P(\mu) x=0 \text { for some nonzero } x \in \mathbb{C}^{n}\right\},
$$

which is closed and contains $\sigma[P(\lambda)]$. The numerical range of matrix polynomials plays an important role in the study of overdamped vibration systems with finite number of degrees of freedom, and it is also related to the stability theory; e.g., see [5,11] and its references. Notice that the notion of $W[P(\lambda)]$ is generalization of the classical numerical range of a matrix $A \in \mathbb{M}_{n}$; namely, $W[\lambda I-A]=W(A)$. In the last 
few years, the generalization of the numerical range of matrices and matrix polynomials has attracted much attention and many interesting results have been obtained; e.g., see $[1-4,15]$. One of these generalizations is the notion of higher numerical ranges. The $k$-numerical range of $P(\lambda)$ is defined and denoted, e.g., see [2], by

$$
W_{k}[P(\lambda)]=\left\{\mu \in \mathbb{C}: \operatorname{tr}\left(X^{*} P(\mu) X\right)=0 \text { for some } X \in \mathcal{X}_{n \times k}\right\} .
$$

Also, the $k-$ spectrum of $P(\lambda)$ is defined as

$$
\sigma^{(k)}[P(\lambda)]=\left\{\mu \in \mathbb{C}: 0 \in \sigma^{(k)}(P(\mu))\right\} .
$$

It is clear that $\sigma^{(k)}[P(\lambda)] \subseteq W_{k}[P(\lambda)]=\left\{\mu \in \mathbb{C}: 0 \in W_{k}(P(\mu))\right\}, \sigma^{(1)}[P(\lambda)]=$ $\sigma[P(\lambda)]$, and $W_{1}[P(\lambda)]=W[P(\lambda)]$. Moreover, if $P(\lambda)=\lambda I-A$, where $A \in \mathbb{M}_{n}$, then $W_{k}[P(\lambda)]=W_{k}(A)$ and $\sigma^{(k)}[P(\lambda)]=\sigma^{(k)}(A)$. The sets $W_{k}[P(\lambda)]$, where $k \in\{1,2 \ldots, n\}$, are generally called the higher numerical ranges of $P(\lambda)$. The joint $k$-numerical range of $P(\lambda)$ is defined as the joint $k$-numerical range of its coefficients; namely, $J W_{k}[P(\lambda)]:=W_{k}\left(A_{0}, A_{1}, \ldots, A_{m}\right)$. It is known, e.g., see [2, Theorem 2.2(iii)], that:

$$
\begin{aligned}
W_{k}[P(\lambda)] & =\left\{\mu \in \mathbb{C}: c_{m} \mu^{m}+\cdots+c_{0}=0,\left(c_{0}, \ldots, c_{m}\right) \in J W_{k}[P(\lambda)]\right\} \\
& =\left\{\mu \in \mathbb{C}: c_{m} \mu^{m}+\cdots+c_{0}=0,\left(c_{0}, \ldots, c_{m}\right) \in \operatorname{conv}\left(J W_{k}[P(\lambda)]\right)\right\} .
\end{aligned}
$$

So, if $Q(\lambda)$ is a matrix polynomial of degree $m$ and arbitrary order such that $J W_{k}[P(\lambda)] \subseteq J W_{k}[Q(\lambda)]$ or if $\operatorname{conv}\left(J W_{k}[P(\lambda)]\right) \subseteq \operatorname{conv}\left(J W_{k}[Q(\lambda)]\right)$, then we have $W_{k}[P(\lambda)] \subseteq W_{k}[Q(\lambda)]$. In the following proposition, we characterize the joint $k$-numerical range of a diagonal matrix polynomial.

Proposition 5. Let $P(\lambda)$, as in (3.1), be a diagonal matrix polynomial. Then $J W_{k}[P(\lambda)]$ is a convex polyhedron. Conversely, every convex polyhedron $H \subseteq$ $\mathbb{C}^{m+1}$ is the joint $k$-numerical range of a diagonal matrix polynomial of degree $m$.

Proof. Suppose that $A_{i}=\operatorname{diag}\left(a_{1}^{(i)}, a_{2}^{(i)}, \ldots, a_{n}^{(i)}\right)$ for $i=0,1, \ldots, m$ and $S_{1}, S_{2}, \ldots, S_{c(n, k)}$ are all the subsets of $\{1,2, \ldots, n\}$ with $k$ elements. By considering $q_{S_{i}}^{(j)}=\frac{\sum_{t \in S_{i}} a_{t}^{(j)}}{k}$ for $j=0,1, \ldots, m$, we have

$$
J W_{k}[P(\lambda)]=\operatorname{conv}\left(\left\{\left(q_{S_{i}}^{(1)}, q_{S_{i}}^{(2)}, \ldots, q_{S_{i}}^{(m)}\right): i=1,2, \ldots, c(n, k)\right\}\right) .
$$

Conversely, suppose that $H \subseteq \mathbb{C}^{m+1}$ is a convex polyhedron with $n$ vertices $v_{1}, v_{2}, \ldots, v_{n}$. Now, we can find the points $\left(c_{i, 0}, c_{i, 1}, \ldots, c_{i, m}\right) \in \mathbb{C}^{m+1}, \quad i=$ $1,2, \ldots, q$ with $q \geq n$ such that their $k$-averages are $v_{1}, v_{2}, \ldots, v_{n}$. Then $H=$ $J W_{k}[D(\lambda)]$, where

$D(\lambda)=\operatorname{diag}\left(c_{1, m}, \ldots, c_{q, m}\right) \lambda^{m}+\cdots+\operatorname{diag}\left(c_{1,1}, \ldots, c_{q, 1}\right) \lambda+\operatorname{diag}\left(c_{1,0}, \ldots, c_{q, 0}\right)$.

So, the proof is complete. 
The following theorem is a generalization of [14, Theorem 2.4].

Theorem 3. Let $P(\lambda)$ be a matrix polynomial as in (3.1). Then

$$
\bigcup W_{k}\left[D_{1}(\lambda)\right]=W_{k}[P(\lambda)]=\bigcap W_{k}\left[D_{2}(\lambda)\right] \text {, }
$$

where the union [intersection] is taken over all diagonal matrix polynomials $D_{1}(\lambda)$ $\left[D_{2}(\lambda)\right]$ of degree $m$ for which $J W_{k}\left[D_{1}(\lambda)\right] \subseteq J W_{k}[P(\lambda)] \subseteq J W_{k}\left[D_{2}(\lambda)\right]$.

Proof. By [1, Theorem 3.1(ii)], the left equality holds.

For the right equality, note that for every diagonal matrix polynomial $D_{2}(\lambda)$ of degree $m$ for which $J W_{k}\left[D_{2}(\lambda)\right] \supseteq J W_{k}[P(\lambda)]$, we have $W_{k}\left[D_{2}(\lambda)\right] \supseteq W_{k}[P(\lambda)]$. Thus

Conversely, let

$$
W_{k}[P(\lambda)] \subseteq \bigcap_{J W_{k}\left[D_{2}(\lambda)\right] \supseteq J W_{k}[P(\lambda)]} W_{k}\left[D_{2}(\lambda)\right] .
$$

$$
\lambda_{0} \in \bigcap_{J W_{k}\left[D_{2}(\lambda)\right] \supseteq J W_{k}[P(\lambda)]} W_{k}\left[D_{2}(\lambda)\right] .
$$

By considering $T_{1}\left(\lambda_{0}\right)=\left\{\left(c_{0}, c_{1}, \ldots, c_{m}\right) \in \mathbb{C}^{m+1}: c_{m} \lambda_{0}^{m}+\cdots+c_{1} \lambda_{0}+c_{0}=0\right\}$, we have $T_{1}\left(\lambda_{0}\right) \cap J W_{k}\left[D_{2}(\lambda)\right] \neq \varnothing$ for every diagonal matrix polynomial $D_{2}(\lambda)$ satisfying $J W_{k}\left[D_{2}(\lambda)\right] \supseteq J W_{k}[P(\lambda)]$. Thus, by [14, lemma 4.1] and Proposition 5, we have

$$
T_{1}\left(\lambda_{0}\right) \cap \operatorname{conv}\left(J W_{k}[P(\lambda)]\right) \neq \varnothing, .
$$

So, by (3.4), $\lambda_{0} \in W_{k}[P(\lambda)]$. Therefore,

$$
W_{k}[P(\lambda)] \supseteq \bigcap_{J W_{k}\left[D_{2}(\lambda)\right] \supseteq J W_{k}[P(\lambda)]} W_{k}\left[D_{2}(\lambda)\right],
$$

and hence, the proof is complete.

Remark 1. In view of Proposition 5 and Theorem 3, one can estimate the shape of the $k$-numerical range of matrix polynomials by convex polyhedrons.

\section{ACKNOWLEDGEMENT}

The authors wish to express their gratitude to anonymous referees for helpful comments and useful suggestions.

\section{REFERENCES}

[1] G. Aghamollaei, N. Avizeh, and Y. Jahanshahi, "Generalized numerical ranges of matrix polynomials." Bull. Iranian Math. Soc., vol. 39, no. 5, pp. 789-803, 2013, doi: -.

[2] G. Aghamollaei and M. Nourollahi, "Higher numerical ranges of matrix polynomials." Bull. Iranian Math. Soc., vol. 41, no. 7, pp. 29-45, 2015, doi: -.

[3] G. Aghamollaei and A. Salemi, "Polynomial numerical hulls of matrix polynomials, II." Linear Multilinear Algebra, vol. 59, no. 3, pp. 291-302, 2011, doi: 10.1080/03081080903436523.

[4] A. Aretaki and J. Maroulas, "On the rank-k numerical range of matrix polynomials." Electronic J. Linear Algebra, vol. 27, no. -, pp. 800-820, 2014, doi: 10.1080/03081080903436523. 
[5] I. Gohberg, P. Lancaster, and L. Rodman, Matrix Polynomials. New York: Academic Press, 1982. doi: 10.1137/1.9780898719024.

[6] H. Green, Matrix Methods in Quantum Mechanics. New York: Barnes-Noble, 1966. doi: -

[7] K. Gustafson and D. Rao, Numerical Range: The Field of Values of Linear Operators and Matrices. $\quad$ New York: Springer-Verlage, 1997. doi: 10.1137/1.9780898719024.

[8] P. Halmos, A Hilbert Space Problem Book. Princeton: D. Van Nostrand, Co., Inc., 1967. doi: - .

[9] R. Horn and C. Johnson, Topics in Matrix Analysis. New York: Cambridge University Press, 1991. doi: -

[10] C. Li, "C-numerical ranges and C-numerical radii." Linear Multilinear Algebra, vol. 37, no. 1-3, pp. 51-82, 1994, doi: 10.1080/03081089408818312.

[11] C. Li and L. Rodman, "Numerical range of matrix polynomials." SIAM J. Matrix Anal. Appl., vol. 15 , no. -, pp. 1256-1265, 1994, doi: -.

[12] C. Li, T. Tam, and N. Tsing, "The generalized spectral radius, numerical radius and spectral norm." Linear Multilinear Algebra, vol. 16, no. 1-4, pp. 215-237, 1984, doi: 10.1080/03081088408817624.

[13] M. Marcus, B. Moyls, and I. Filippenko, "Normality and the higher numerical range." Can. J. Math., vol. 30, no. 1-4, pp. 419-430, 1978, doi: 10.4153/CJM-1978-036-6.

[14] P. Psarrakos and M. Tsatsomeros, "On the relation between the numerical range and the joint numerical range of matrix polynomials.” Electronic J. Linear Algebra, vol. 6, no. -, pp. 20-30, 2000, doi: 10.1080/03081080903436523.

[15] M. Zahraei and G. Aghamollaei, "Higher rank numerical ranges of rectangular matrices." Ann. Funct. Anal., vol. 6, no. 2, pp. 133-142, 2015, doi: 10.15352/afa/06-2-12.

Authors' addresses

Gholamreza Aghamollaei

Department of Pure Mathematics, Faculty of Mathematics and Computer, Shahid Bahonar University of Kerman, Kerman, Iran

E-mail address: aghamollaei@uk.ac.ir, gh_aghamollaei@yahoo.com

Mohammad Ali Nourollahi

Department of Mathematics, Higher Education Complex of Bam, Bam, Kerman, Iran

E-mail address: mnourollahi@yahoo.com, mnourollahi@bam.ac.ir 